\title{
The significance of antineutrophil cytoplasmic antibody in microscopic polyangitis and classic polyarteritis nodosa
}

\author{
A Bakkaloglu, S Ozen, E Baskin, N Besbas, A Gur-Guven, O Kasapçopur, K Tinaztepe
}

\begin{abstract}
Aims-To describe the distribution and features of classic polyarteritis nodosa (PAN) and microscopic polyarteritis (MPA) and the importance of antineutrophil cytoplasmic antibody (ANCA) in childhood PAN.

Methods-Classic PAN was diagnosed in 15 patients based on the presence of aneurysms on angiography in 10 patients and of necrotising vasculitis in medium sized arteries in five. MPA was diagnosed in $\mathbf{1 0}$ patients, based on characteristic findings at renal biopsy in six and by the presence of small sized necrotising arteritis in four. Serum ANCA was detected initially by indirect immunofluorescence (IIF) followed by an immunoassay for myeloperoxidase (MPO) in each case.
\end{abstract}

Results-The median age of the patients with classic PAN and MPA was 12 (range 8-17) and 9.5 (range 5-14) respectively. None of the patients with classic PAN had renal failure. Six of the patients with MPA presented with renal failure; four progressed to chronic renal failure. Clinically evident pulmonary-renal syndrome was present in three of the 10 patients with MPA. IIF for ANCA in classic PAN was negative in nine, showed mild staining patterns in six, and in one MPO-ELISA was mildly increased. IIF for ANCA in MPA revealed very strong perinuclear ANCA staining in nine and atypical staining in one. In MPA, median MPO-ELISA level was $42.5 \mathrm{EU} / \mathrm{ml}$ (range 20-250). Treatment of childhood PAN was satisfactory with effective treatment; however relapses did occur.

Conclusion-ANCA is useful in the diagnosis and follow up of MPA.

(Arch Dis Child 2001;85:427-430)

Keywords: microscopic polyarteritis; classic polyarteritis; ANCA

Polyarteritis nodosa (PAN) is a heterogeneous group of vasculitides which have been subclassified into microscopic and classic forms. ${ }^{12}$ Predominant small vessel involvement is the diagnostic criterion of microscopic polyarteritis (MPA); predominant medium sized vessel involvement defines classic PAN. Both are rare in childhood. MPA and classic PAN differ in clinical features and outcome, and have different antineutrophil cytoplasmic antibody (ANCA) specificities. ANCA has been reported to be unusual in classic PAN. ${ }^{3-6}$
ANCA is important, especially for the diagnosis and follow up of MPA and Wegener's granulomatosis. ${ }^{78}$ However, the autoantibody is directed against different neutrophil constituents in MPA and Wegener's granulomatosis. ANCA is classified into two main groups according to the fluorescence appearance on ethanol fixed neutrophil preparations: perinuclear (p) ANCA and cytoplasmic (c) ANCA. In MPA, pANCA is seen and myeloperoxidase (MPO) is the principal target antigen. ${ }^{9}$ ANCA is thought to contribute to the immunoinflammatory process in these vasculitides. On the other hand ANCA is only very rarely seen in reported cases of classic PAN. ${ }^{10}$

We present a series of childhood cases of MPA and classic PAN, emphasising the association with ANCA and other specific features of these patients.

\section{Patients and methods}

Twenty two patients referred to our department between 1990 and 1999 and three further cases from two other paediatric departments (each with a positive MPO-ANCA titre) are described. All patients met the clinical diagnostic criteria for PAN. ${ }^{11}$ Patient follow up was 1-9 years (median 6 years). Classic PAN was diagnosed in 15 patients by the demonstration of aneurysms on angiography in 10 , and by the presence of necrotising vasculitis in mainly medium sized arteries in five patients. MPA was diagnosed in a total of 10 patients: six had characteristic findings on renal biopsy and four had necrotising arteritis in non-renal small arteries. Only patients who underwent immunofluorescence (IIF) and enzyme linked immunosorbent assay (ELISA) MPO-ANCA studies were included.

Routine laboratory tests for complete blood counts and acute phase reactants, renal function tests, urinalysis, and determination of ANCA in serum were performed in all patients.

ANCA were initially studied by the indirect immunofluorescence method. ANCA determination by IIF was performed using commercial substrate plates on $1 / 20$ diluted sera using ethanol fixed and subsequently formalin fixed slides (INOVA). Sera positive by IIF were subsequently tested by ELISA kits (EuroDiagnostica) for MPO-ANCA. An increased titre was defined as greater than $20 \mathrm{EU} / \mathrm{ml}$.

Renal biopsy was performed in six patients who had clinical findings of glomerular involvement. Biopsy specimens were obtained from peripheral nerve tissue in one, nodules in two, intestinal tissue in two, and skin and subcutaneous tissue in five patients. 
Table 1 Summary of clinical findings in patients with MPA and classic PAN

\begin{tabular}{lll}
\hline & $\begin{array}{l}M P A(\%=10) \\
(\%)\end{array}$ & $\begin{array}{l}\text { Classic PAN }(n=15) \\
(\%)\end{array}$ \\
\hline Sex (F/M) & $6 / 4$ & $8 / 7$ \\
Median age (y) (range) & $12(8-17)$ & $9.5(5-14)$ \\
Constitutional manifestations & $10(100)$ & $13(86.7)$ \\
Myalgia & $2(20)$ & $12(80)$ \\
Skin lesions & $7(70)$ & $8(53.3)$ \\
Joint disease & $6 / 9(66.7)$ & $7(46.7)$ \\
Gastrointestinal symptoms & $2 / 9(22.2)$ & $2(13.3)$ \\
Neurological findings & $2 / 9(22.2)$ & $5(33.3)$ \\
Pulmonary findings & $3(30)$ & 0 \\
Hypertension & $5(50)$ & $3(53)$ \\
Haematuria & $5(50)$ & $3(20)$ \\
Proteinuria* & $7(70)$ & 0 \\
Renal failure (Cr Cl<\%50) & $6(60)$ & - \\
End stage renal failure & $4(40)$ & $2(20)$ \\
Relapses & $5(50)$ & \\
\hline
\end{tabular}

${ }^{\star} \mathrm{p}<0.05$.

Genetic analysis for familial Mediterranean fever (FMF) mutations was performed in one patient who had typical features of FMF. For mutation analysis of this gene, exon 10 , which harbours 14 mutations, was sequenced by DGGE and E148Q was analysed by restriction endonuclease enzyme (Bst NI) digestion.

Treatment and disease course were evaluated retrospectively. Clinical remission was defined as complete resolution of the clinical symptoms together with normal values of acute phase reactants. Any clinical and laboratory flare up was defined as a relapse of the vasculitis. Chronic renal insufficiency was defined as a glomerular filtration rate less than $50 \mathrm{ml} / \mathrm{min} /$ $1.73 \mathrm{~m}^{2}$. End stage renal failure was defined as a glomerular filtration rate less than 10 $\mathrm{ml} / \mathrm{min} / 1.73 \mathrm{~m}^{2}$.

For statistical analysis, Mann-Whitney $U$ and $\chi^{2}$ tests were used to compare data. Probability values less than 0.05 were considered significant.

\section{Results}

Table 1 summarises clinical features. During the study period none of the patients died. Constitutional symptoms (fever, weight loss, and fatigue) were present in all of the MPA patients and in $86.7 \%(13 / 15)$ of the classic PAN patients. Myalgia was present significantly more frequently in patients with classic

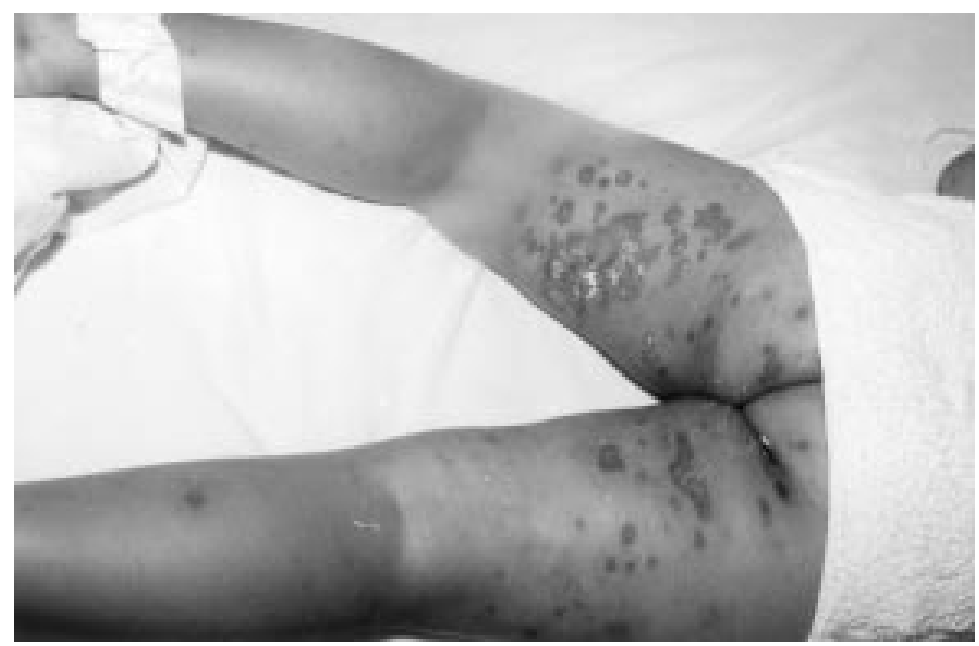

Figure 1 Necrotising vasculitis of the skin in a patient with a high MPO-ANCA titre.
PAN than in patients with MPA. Figure 1 shows typical skin lesions.

Proteinuria was more common in the MPA group as was renal failure (six presented with this, of whom four progressed to chronic renal insufficiency within three to six months). One has received a successful renal transplant from the father and remains well one year later. The others are currently on chronic dialysis therapy.

Clinically evident pulmonary-renal syndrome was present in three of the six MPA patients (aged 9, 10, and 12 years, respectively; two boys) who had renal failure. They presented with severe constitutional symptoms and rapidly progressive glomerulonephritis. All had to be dialysed for acute renal failure, progressed to renal insufficiency, and started chronic dialysis treatment within one to five years. They also had pulmonary symptoms at presentation and pulmonary involvement was confirmed by pulmonary perfusion scans. These three patients had the highest MPOANCA levels ranging from 150 to $250 \mathrm{EU} / \mathrm{ml}$. The other three MPA patients had necrotising glomerulonephritis and presented with acute renal failure. Only one has developed chronic renal insufficiency. One of these MPA patients also had hypertension. A renal angiogram revealed aneurysms in the renal artery. His serum hepatitis B surface antigen was positive. He had a clinical diagnosis of FMF because of his recurrent fever and abdominal pain attacks, subsequently confirmed by demonstration of M694V homozygosity. He entered remission with immunosuppressive treatment but relapsed with complaints of double vision. Magnetic resonance imaging scan showed central nervous system vasculitis. $\mathrm{He}$ also developed severe gastrointestinal and musculoskeletal system involvement. Interestingly, the MPOANCA was only mildly raised (borderline) in this patient.

One other child with MPA had severe gastrointestinal tract vasculitis, another had nervous system abnormalities (optic neuritis), and a third had peripheral nervous system findings. One girl who had very severe skin vasculitis with widespread necrosis and hypertension, had necrotising vasculitis in small vessels on skin biopsy. A renal angiogram was normal. She also had arthritis and myalgia. Her clinical course was characterised by remissions and exacerbations of disease activity, and she experienced a major relapse after a local earthquake in 1999. Her serum MPO-ANCA was one of the highest with a titre of $200 \mathrm{EU} / \mathrm{ml}$.

Of the 15 patients with classic PAN, eight presented with hypertension and were diagnosed by renal angiography (table 1 ). Five were diagnosed with medium sized artery involvement in biopsy specimens from subcutaneous tissue and/or muscle. None had small sized artery involvement. Two had relapses in their clinical course. None had hepatitis B surface antigenaemia.

All patients had raised erythrocyte sedimentation rate and $\mathrm{C}$ reactive protein levels at the time of diagnosis (table 2). Among children with classic PAN, IIF for ANCA was negative in nine, showed a weak c-ANCA pattern in five, 
Table 2 Laboratory findings in patients

\begin{tabular}{llll}
\hline Test & $\begin{array}{l}\text { MPA }(n=10) \\
\text { median; range }(\%)\end{array}$ & $\begin{array}{l}\text { Classic PAN }(n=15) \\
\text { median; range (\%) }\end{array}$ & p value \\
\hline $\begin{array}{l}\text { Erythrocyte sedimentation rate }(\mathrm{mm} / \mathrm{h}) \\
\text { C reactive protein }(\mathrm{mg} / \mathrm{dl})\end{array}$ & $85 ; 22-128$ & $48 ; 28-104$ & $>0.05$ \\
Urea $(\mathrm{mg} / \mathrm{dl})$ & $25.5 ; 2.9-76$ & $19.2 ; 0.5-48$ & $>0.05$ \\
Creatinine $(\mathrm{mg} / \mathrm{dl})$ & $39 ; 14-78$ & $14 ; 9-19$ & $<0.05$ \\
MPO-ANCA (EU/ml) & $2.6 ; 0.4-7.8$ & $0.5 ; 0.3-0.8$ & $<0.05$ \\
High MPO-ANCA levels & $42.50 ; 20-250$ & $5.0 ; 5-30$ & \\
Indirect immunofluorescence staining for ANCA & $10(100)$ & $1(6.7)$ & \\
$\quad$ Negative & 0 & & \\
c-ANCA pattern & 0 & $9(60)$ & \\
p-ANCA pattern & $9(90)$ & $5(33)$ & \\
Atypical & $1(10)$ & $1(6.7)$ & \\
\hline
\end{tabular}

and p-ANCA in one. However, MPO-ELISA was negative in all but one case. In contrast, among children with MPA, IIF for ANCA revealed very strong $\mathrm{p}$-ANCA staining in nine and atypical staining in one (table 2). All patients with pulmonary and renal involvement had very strong fluorescence with a p-ANCA pattern and had raised MPO-ANCA titres.

In one pateint with MPA who presented with renal and pulmonary involvement, we measured MPO-ANCA levels on three occasions. The high level at presentation decreased notably with remission and rose again on relapse.

All patients were treated with corticosteroids and oral cyclophosphamide. After three months, cyclophosphamide was changed to azathioprine. In patients with MPA, corticosteroids were given intravenously at a dose of $10-30 \mathrm{mg} / \mathrm{kg} /$ day for three consecutive days. Corticosteroid treatment was reinstituted for relapses. The route and dosage varied between patients.

\section{Discussion}

In this case series we compare MPA and classic PAN, and their association with ANCA. The diagnosis of these patients was based on the Chapel Hill consensus criteria.

Several previous childhood series have not distinguished clearly between MPA and classic PAN. ${ }^{12-15}$ Series in children reporting ANCA are also rare. Ellis et al reported five in 1995, ${ }^{16}$ concluding that pulmonary involvement is less common in children. Among their five patients, four had acute renal failure at onset, of whom three developed end stage renal disease. They reviewed six other reported ANCA associated childhood patients. Of the 11, seven had c-ANCA, whereas only four had p-ANCA. They concluded that patients with ANCA tended to have rapid renal deterioration, and that c-ANCA positivity, suggestive of Wegener's disease, may predict bad outcome. Sediva et al reported ANCA studies in a series of children, none of whom had PAN. ${ }^{17}$ Valentini et al reported a series of seven children with ANCA associated kidney disease. ${ }^{18}$ By IIF, four were c-ANCA positive, and three were p-ANCA positive but ELISA studies were not available. At presentation five had renal insufficiency. End stage renal disease developed in one and a further two developed renal insufficiency during a short follow up period. Only one developed systemic features.
Among our 25 patients, three had full blown pulmonary-renal syndrome with very high MPO-ANCA titres. All progressed to end stage renal disease. High MPO-ANCA levels at presentation may therefore be associated with a poor prognosis.

Rapidly progressive glomerulonephritis is the distinguishing feature of MPA..$^{19}$ Six of our patients had this typical presentation. Another four were classified as MPA, based on the presence of necrotising vasculitis of predominantly small vessels. The adult series reported by Guillevin et al also described renal manifestations in only 37 of 47 patients. ${ }^{19}$ However, the clinical features and the renal course of patients without glomerulonephritis are quite different.

The IIF pattern and ELISA for MPO were very useful in the differentiation between MPA and classic PAN. A p-ANCA pattern by IIF was strongly suggestive of MPA, although it was present in one classic PAN patient as well, as was an increased MPO-ANCA level (again mildly increased in only one patient with classic PAN). The one patient with MPA with borderline MPO-ANCA titres had overlapping clinical features of both MPA and classic PAN with renal aneurysms, frank systemic symptoms, and central nervous system involvement. We suggest that it is not possible to differentiate fully between MPA and classic PAN in cases such as this. FMF may also have complicated the course of this case. The problems with classification have been previously addressed by other authors and by the Chapel Hill group as well. ${ }^{1} 320$

Although we have not performed ELISA tests for other ANCA antigens, all our patients with a c-ANCA staining pattern had aneurysms that are typical for classic PAN. Among the clinical features, only renal failure and myalgia were significantly different between those with MPA and classic PAN in this series. The renal prognosis is guarded in patients with acute renal failure at presentation. The relapse rates were not significantly different between the two groups but numbers were small. In one patient the relapsing course was associated with the earthquake. Psychological and/or physicochemical factors might have affected her disease course. Yashiro et al have recently reported higher morbidity in their ANCA related angiitis and/or nephritis patients after the 1995 earthquake in Japan. ${ }^{21}$ They suggested that there might be a relation with disease development as a result of air pollution affecting respiratory tract inflammation.

In conclusion, when diagnosed promptly, the treatment of childhood PAN is satisfactory and there was no mortality in the presented series. However, long term follow up is necessary as severe relapses do occur. In the presented childhood series, classic PAN was more common than MPA. We suggest that ANCA is an important serological marker for the diagnosis of MPA and is associated with more severe kidney disease. 
This study was supported by The Turkish Scientific and Technical Research Council ("TUBITAK"), project number TAG1211.

1 Jennette JC, Falk RJ. Overview of the nomenclature and dennette JC, Falk RJ. Overview of the nomenclature and diagnostic catego

2 Jennette JC, Falk RJ, Andrassy K, et al. Nomenclature of systemic vasculitides; the proposal of an international consensus conference. Arthritis Rheum 1994;37:187-92.

3 Guillevin L, Lhote F. Classification and management of necrotising vasculitides. Drug 1997;53:805-16.

4 Athreya B. Vasculitis in children. Pediatr Clin $N \mathrm{Am}$ 1995;42:1239-61.

5 Guillevin L, Lhote F, Amouroux J, et al. Antineutrophil cytoplasmic antibodies, abnormal angiograms and pathological findings in polyarteritis nodosa and Churg-Strauss syndrome: indications for the classification of vasculitides of the polyarteritis nodosa group. Br 7 Rheumatol 1996;35: 958-64.

6 El-Reshaid K, Kapoor MM, El-Reshaid W, et al. The spectrum of renal disease associated with microscopic polyangiitis and classic polyarteritis nodosa in Kuwait. Nephrol Dial Transplant 1997;12:1874 82 .

7 Jennette JC, Wilkman AS, Falk RJ. Diagnostic predictive value of ANCA serology. Kidney Int 1998;53:796-8.

8 Hagen CH, Daha MR, Hermans J, et al. For the EC/BCR Hagen $\mathrm{CH}$, Daha MR, Hermans J, et al. For the EC/BCR
project for ANCA assay standardization. The diagnostic project for ANCA assay standardization. The diagnostic
value of standardized assays for anti-neutrophil cytoplasmic antibodies (ANCA) in idiopathic systemic vasculitis. Kidney Int 1998;53:743-53.

9 Hoffman GS, Specks U. Antineutrophil cytoplasmic antibodies. Arthritis Rheum 1998;41:1521-37.

10 Guillevin L, Visser H, Noel LH, et al. Antineutrophil cytoplasmic antibodies in systemic polyarteritis nodosa with and without hepatitis B virus infection and Churg-Strauss syndrome-62 patients. F Rheumatol 1993;20:1345-9.
11 Ozen S, Besbas N, Saatci U, Bakkaloglu A. Diagnostic criteria for polyarteritis nodosa in childhood. If Pediatr $1992 ; 2: 206-9$

12 Gur H, Tchakmakjan I, Eherenfeld M, Sidi Y. Polyarteritis nodosa: a report from Israel. Am f Med Sci 1999;317:23842 .

13 Maeda M, Kobayashi M, Okamoto S, et al. Clinical observation of 14 cases of childhood polyarteritis nodosa in Japan. Acta Paediatr fpn 1997;39:277-9

14 Kumar L, Sarkar B, Singh S, et al. Polyarteritis nodosa-a few unusual findings. Indian Pediatr 1996;33:459-64.

15 Magilavy DB, Petty RE, Cassidy JT, Sullivan DB. A syndrome of childhood polyarteritis. F Pediatr 1977;91:2530.

16 Ellis EN, Wood EG, Berry P. Spectrum of disease associated with anti-neutrophil cytoplasmic antibodies in pediatric patients. F Pediatr 1995;126:40-3.

17 Sediva A, Kolarova I, Bartunkova J. Antineutrophil cytoplasmic antibodies in children. Eur F Pediatr 1998;152: 986-91.

18 Valentini RP, Smoyer WE, Sedman A, et al. Outcome of antineutrophil cytoplasmic antibodies-positive glomerulonephritis and vasculitis in children: a single center nephritis and vasculitis in childre
experience. F Pediatr 1998;132:325-8.

19 Guillevin L, Durand-Gasselin B, Cevallos R, et al. Microscopic polyangiitis: clinical and laboratory findings in eighty-five patients. Arthritis Rheum 1999;42:421-30.

20 Bosch X. Microscopic polyangiitis (microscopic polyarteritis) with late emergence of generalised Wegener's granulomatosis. Ann Rheum Dis 1999;58:644-7.

21 Yashiro M, Muso E, Itoh-Ihara $\mathrm{T}$, et al. Significantly high regional morbidity of MPO-ANCA related angiitis and/or nephritis with respiratory tract invovlement after the 1995 great earthquake in Kobe. Am f Kid Dis 2000; 35:889-95.

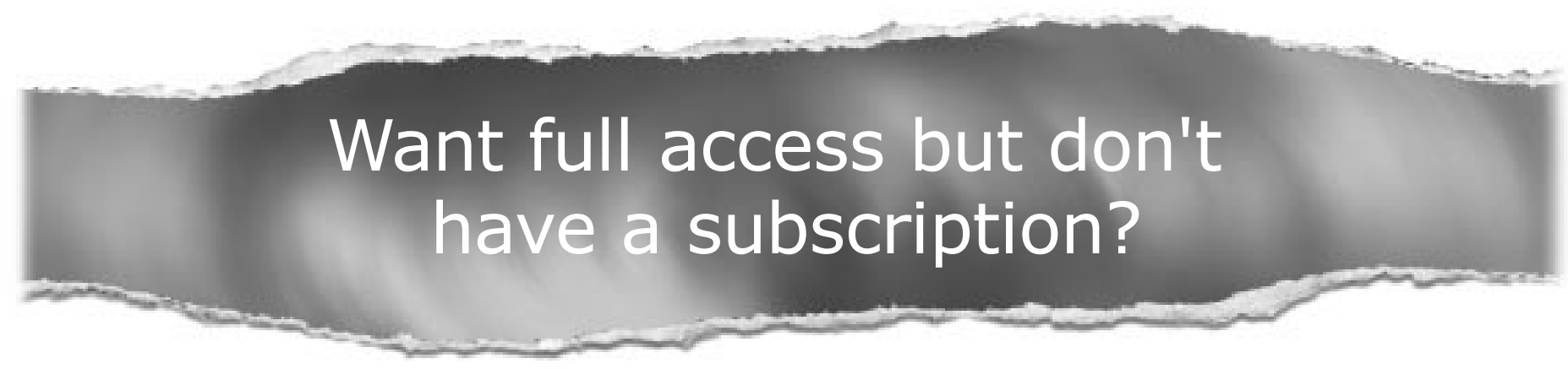

\section{Pay per access}

For just US $\$ 25$ you can have instant access to the whole website for 30 days. During this time you will be able to access the full text for all issues (including supplements) available. You will also be able to download and print any relevant pdf files for personal use, and take advantage of all the special features Archives of Disease in Childhood online has to offer.

\section{www.archdischild.com}

